\title{
Über das Züchten der Larven der gewöhnlichen Fleischfliege (Calliphora vomitoria) in sterilisierten Nährmitteln.
}

Von

\author{
E. A. Bogdanow, \\ Dozent am Moskauer landw. Institute.
}

(Mit 3 Textfiguren.)

In dem unlängst erschienenen Heft der Zeitschrift fürBiologie (Bd. 47, Heft 2. 1905) veröffentlicht $\mathrm{E}$. We in land seine sehr interessanten Untersuchungen über den Stoffwechsel der gewöhnlichen Fleischfliege (Calliphora vomitoria). ${ }^{\text {) }}$ Die Beobachtungen, welche von dem genannten Forscher gemacht wurden, berühren ziemlich nahe die Erscheinungen im Dipteren-Leben, welche ich seit längerer Zeit (nämlich seit 1898, aber besonders in den Jahren 1902-1905) untersucht babe. Da ich einige wichtige Angaben bestätigen, andere in mancher Hinsicht vervollständigen kann, so scheint es mir wünschenswert, das von mir bis jetzt Gewonnene in kurzer Darstellung zusammenzufassen. Dieser kurze Bericht sollte schon am Ende des vorigen Jahres erscheinen, aber während der blutigen Erscheinungen im Leben der russischen Gesellschaft fand ich nieht die dazu nötige Kraft.

Die Physiologie der Fliegenlarve interessierte mich nicht ihrer selbst wegen, sondern wegen ihrer nahen Beziehung zu dem Thema, mit welchem ich mich hauptsächlich beschäftigte - nämlich zur viel diskutierten Frage über die Entstehung von Fett aus Eiweiss. Es ist bekannt, dass die Untersuchungen ron $\mathrm{H}$ of $\mathrm{m}$ a $\mathrm{n} \mathrm{n}$ über die Fettbildung bei Fliegenmaden (Zeitschr. f. Biologie 8) lange Zeit einen der wichtigsten Stützpunkte dieser Lehre bildeten, verloren aber den

1) Ernst Weinland, Über die Stoffumsetzungen während der Metamorphose der Fleischfliege (Calliphora vomitoria). - E. Weinland, Über die Ausscheidung von Ammoniak durch die Larven von Calliphora und über eine Beziehung dieser Tatsache zu dem Entwicklungsstadium dieser Tiere.

E. Pflüger, Archiv für Physiologie. Bd. 113. 
grössten Teil ibres Interesses, seitdem sie von Prof. Pflüger einer ernsten Kritik unterworfen wurden (Pflüger's Archiv 51, S. 229). Für meine Hauptversuche wählte ich als Versuchsobjekt Ferkel aus (die dabei gewonnenen Resultate sollen in der nächsten Zeit veröffentlicht werden); es schien mir aber angebracht, auch die von Hofmann zum ersten Male untersuchte Fliegenlarve nochmals zu prüfen, freilich bei ganz anderer Anordnung der Versuche. Die von mir allmählich ausgearbeiteten Methoden der Züchtung will ich hier kurz beschreiben. Der schwächste Punkt der Hofmann'schen Untersuchungen lag bekanntlich darin, dass er seine Fliegenlarven in einer sich in reger Zersetzung befindenden Substanz (nämlich im Blute) züchtete, so dass man die von den Bakterien in der Nahrung gebildeten Zersetzungsprodukte als eigentliche Quelle der von ihm beobachteten Fettproduktion ansehen konnte. Es lag darum sehr nahe, dieselben Larven bei einer solchen Versuchsanordnung zu züchten, bei welcher die Bakterientätigkeit zum grössten Teil oder auch vollständig ausgeschlossen werden konnte. Es war dazu aber eine grosse Anzahl von Vorversuchen nötig, weil das Züchten von tierischen Organismen unter solchen Bedingungen. wenn auch möglich, zu den schwierigsten Aufgaben gezählt werden darf. Meine ersten grösseren Versuche (1898) haben mir schon ziemlich brauchbare Resultate geliefert. Es hat sich herausgestellt, dass die Fliegenlarven, wenn auch nicht immer, so doch zuweilen eine ansehnliche Grösse erreichen können, wenn man ihnen ausschliesslich frisches Hühnereiweiss oder ca. 10\% Albumosenlösung („Pepton“ nach Adamkiewicz) zur Verfügung stellt und jeden Tag die Nahrung erneuert. Ich habe zu diesem Zwecke platte Glasschalen (fast gewöhnliche Petri'sche Schalen, doch ziemlich hohe) gebraucht. Damit die Eier und Larven in der Flüssigkeit nicht untersinken, legte ich zunächst in die Schalen genügende Mengen Baumwolle, später, was noch besser ist, Glaswolle, welche sich vorzüglich zum Einsaugen der Flüssigkeit eignete (und auch jeden Tag erneuert wurde) ${ }^{1}$ ). Es stellte sich dabei heraus, dass vollständig frische Nahrung, zum Beispiel gekochtes und gehacktes Fleisch, die Larven weniger anlockt als dieselbe Substanz, wenn sie aus einem Rest der Larvennahrung besteht; es schien mir auch, dass jede Erneuerung der Eiweiss-

1) Damit die Larven aus der Schale nicht entfliehen können, belegte ich gewöhnlich den Schalendeckel mit Gipsbrei herum. 
lösung den Larven im Anfang unangenehm war. Ich bemerke auch dabei, dass es nicht immer, vielmehr selten gelingt, die Larven bei Anwendung derselben Eiweissnahrung grosszuziehen.

Aus diesen Beobachtungen habe ich den Schluss gezogen, dass gewisse, von den Mikroorganismen verursachte Zersetzungen eine unumgängliche Bedingung für die ungestörte Ernährung der Larve seien. Einen weiteren Schritt vorwärts machte ich fast zufällig, indem ich in einer anderen Reihe von grösseren. Versuchen im Sommer 1902 die Entwicklung der Larven in Eiweisslösungen mit dem Zusatze verschiedener Reagenzien und zuweilen auch Farbstoffe beobachtete. In einigen Mischungen wollten die Larven überhaupt nicht wachsen; als ich eine kleine Menge Ammoniaklösung zusetzte, ging die Entwicklung eine Zeitlang rasch vorwärts. Aus solchen vergleichenden Beobachtungen ging es unzweifelhaft hervor, dass zu den Vorbedingungen der Larvenentwicklung vor allen Dingen Ammoniakbildung gehört.

Ich gehe jetzt über zu meinen Versuchen über das Züchten der Fliegenlarven in vollkommen sterilisierten Nährmitteln. Schon im Jabre 1898 stellte ich im bakteriologischen Laboratorium des Moskauer Landwirtschaftlichen Instituts einige Versuche dieser Art an, bei welchen ich verschiedenen Ratschlägen über die Untersuchungstechnik von seiten des Vorstandes des genannten Laboratoriums, Prof. N. Chudiakow, folgen konnte. Die dabei gewonnenen Resultate waren keineswegs ermunternder Art, wahrscheinlich deshalb, weil jch nur Albumosenlösungen ohne Ammoniakzusatz probierte; alle Larven starben fast in den ersten Entwicklungsstadien. Viel mehr wurde in den Jahren 1902-1905 gewonnen, indem auch die Technik der Untersuchung manchen Verbesserungen unterworfen wurde. Die Fliegeneier wurden zunächst mit der wässerigen Sublimatlösung (5: 1000) sterilisiert, wobei die Entwicklungsfähigkeit in den meisten Fälleu keine Beeinträchtigung erfährt. ${ }^{1}$ ) Es geschieht dies am besten, wenn man ein wenig Glaswolle in Form einer Platte ausbreitet und mit destilliertem Wasser übergiesst; aus dieser Platte schneidet man ein längliches Viereck aus und in die Mitte legt man die Fliegeneier eins nach dem andern (damit sie keinen Klumpen bilden und von allen Seiten von der Sublimatlösung umspült werden können). Ich nahm gewöhnlich 70-100 Eier zu jedem Versuch. Wenn alle

1) Die unlängst von der Fliege gelegten Eier scheinen das nicht zu ertragen. 
Eier bequem verteilt waren, rollte ich das Viereck wie ein Rohr zusammen, befestigte es in dieser Lage mit Hilfe eines kurzen Fadens; in beide seitlichen Öffnungen legte ich ausserdem kleine, von derselben Glaswollenplatte abgeschnittene Stückchen, damit möglichst wenige Eier beim Sterilisieren und Nachwaschen verloren gehen. Das beschriebene Rohr mit den Fliegeneiern wurde rasch in einen dazu konstruierten und vorher sterilisierten einfachen Apparat

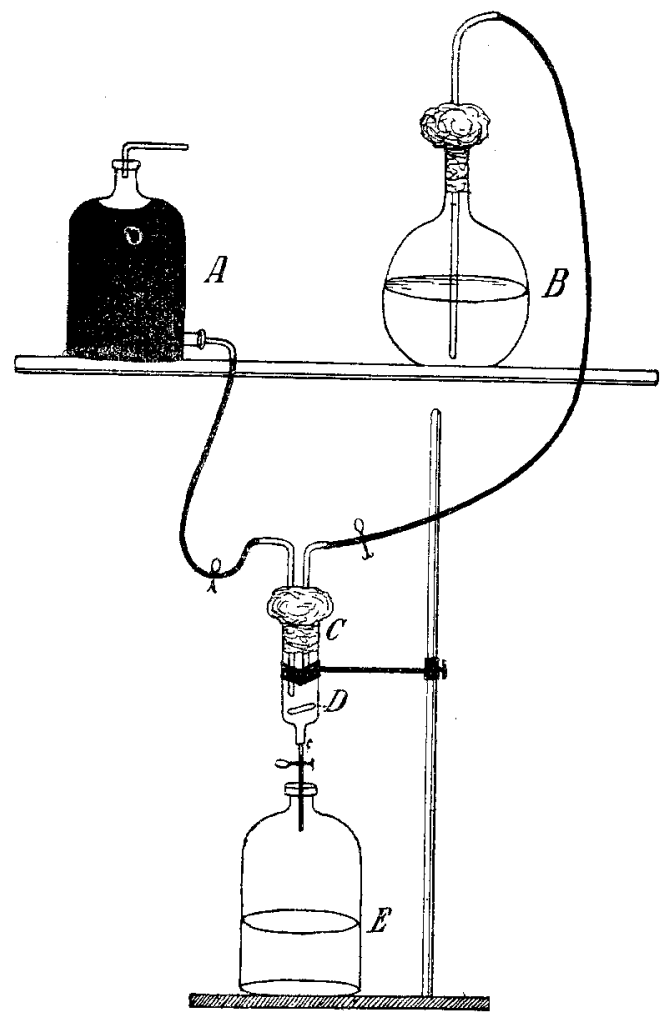

Fig. 1. Apparat zum Sterilisieren der Eier. A Flasche mit Sublimatlösung. $B$ Flasche mit destilliertem Wasser. $C$ Glasrohr, in welchem Glaswolle $(D)$ mit den Eiern sterilisiert wird. $E$ Flasche mit Waschwasser.

gebracht, welcher aus einer $12 \mathrm{~cm}$ langen und $4^{1 / 2} \mathrm{~cm}$ breiten (im Diameter), unten sehr verengten Glasröhre bestand, welche mit einem Baumwollpfropfen und mit zwei durch denselben durchgehenden, knieförmig gebogenen Röbrchen (einem langen und einem kurzen) versehen war. Die Röhrchenenden wurden wie gewöhnlich mit kleinen Baumwollstückchen vor Bakterien geschützt; unten endigte die Röhre mit einem Gummischlauch mit Quetschhahn und kleinem Glasstäbchen. 
Das längere von den genannten Röhrchen wurde nun mit einer Sublimatflasche mittelst eines Käutschukrohres vereinigt, wobei die gewöhnlichen Vorsichtsmassregeln nicht ausser achtgelassen wurden; das kürzere Röhrehen vereinigte man mit dem grossen Kolben, der destilliertes und sterilisiertes Wasser enthielt. Jetzt wurde der untere Teil der Glasröhre mit der.Sublimatlösung gefült und die Eier gewöhnlich $1^{1} / 2$ Minuten darin gelassen unter fortwährendem Schütteln; dann liess ich die Sublimatlösung ablaufen und wiederholte das Sterilisieren noch $1^{1 / 2}$ Minuten lang (also insgesamt 3 Minuten). Dann wurden die Eier mit dem sterilisierten, destillierten Wasser gut ausgewaschen (gewöhnlich bis zu $1^{1 / 2}$ Liter Waschwasser). Die

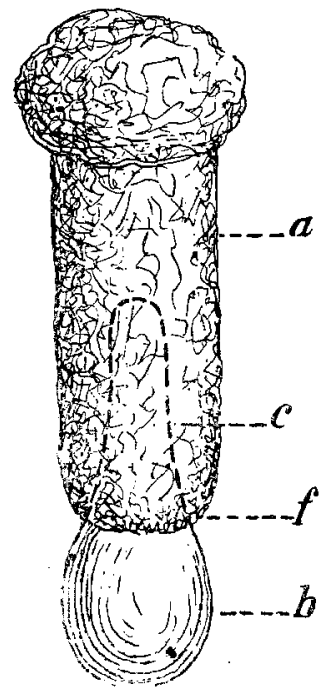
Pfropfens für Reagenzglas mit der Nahrung. $a$ Baumwolle, $b$ Glaswolle, $c$ der obere Teil der Glaswolle in Projektion, $f$ Fäden, welche Glaswolle mit der Baumwolle vereinigen.
Fig. 2. Schematische Darstellung eines

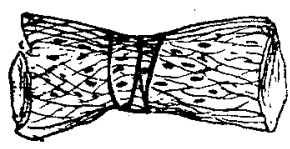

Fig. 3. Schematische Darstellung eines Rohres aus Glaswolle mit den Eiern.

beschriebene Methode gestattet, die Sterilisation jedesmal als vollständig zu betrachten; das wurde einige Male noch besonders bestätigt, als die Eier in die mit Nährgelatine gefüllten Petrischen Schalen gebracht, gequetscht und einige Zeit lang stehen gelassen wurden. Nach der erfolgten Sterilisation sollten die Eier in die passende sterilisierte Nahrung gebracht werden. Als bequeme Nahrungsbehälter wurden zuletzt nur noch lange Reagenzgläser ${ }^{1}$ ) gebraucht und in folgender Weise gefüllt. Ich wog zuerst eine genügende Menge von Fleisch oder anderer geeigneter Substanz ${ }^{2}$ ) ab (Kasein, Eieralbumin, Albumosen usw.) und setzte gewöhnlich eine 
geringe Quantität Ammoniaklösung binzu ${ }^{1}$ ) (nämlich 5 ccm, ca. $1 \%$ ige Lösung und 2-3 Tropfen der $20 \%$ igen Lösung), rührte alles gut um und füllte damit die Reagenzgläser. Als Verschluss diente ein Klumpen Glaswolle, welcher von allen Seiten, exklusive von unten, mit Baumwolle umgeben und so (mit Hilfe der Fäden) eingerichtet war, dass er bequem in einem Zuge herausgezogen und wieder eingesetzt werden konnte. Es gehört viel Übung dazu, einen solchen Pfropfen gut darzustellen, er ist aber sehr nützlich, indem er die grösseren Larven hindert, in die Baumwolle hineinzukriechen, was sie gern tun. Das ganze wurde im bakteriologischen Laboratorium in gehöriger Weise sterilisiert.

Das Übertragen der sterilisierten Eier in die mit Nahrung gefüllten Reagenzgläser und das Aufbewahren derselben geschah in einem dazu speziell aus Holz und Glas konstruierten Kasten, welcher aussen und innen mit Vaselin bestrichen war; auf diese Weise wurden meine "Kulturen" vor Staub vorzüglich geschützt. Wenn alles in der soeben beschriebenen Weise gut eingerichtet ist, geht die Entwicklung der Larven in einem gewissen Prozentsatze der Fälle eine Zeitlang ziemlich gut, um später aber, und meistens, wenn sie die Grösse von ungefähr $0,9 \mathrm{~cm}$ Länge und $0,15 \mathrm{~cm}$ Breite erreichen, stillzustehen. Die Larven hören nämlich auf zu fressen, sehen sehr mager und durchsichtig aus. Durch die bis jetzt unbekannten individuellen Verschiedenheiten der Larven geschieht es, dass in ein und derselben Nahrung einige von ihnen sterben, andere aber gut gedeihen; es spielt dabei zuweilen eine gewisse Rolle, dass die stärkeren Larven die schwächeren fressen. Einmal habe ich (nämlich im Kasein) nur eine von hundert Larven gross gezogen, alle anderen starben sehr bald, diese aber wurde ganz besonders gross und fett. Die oben ziemlich ausführlich angeführte Beschreibung der Sterilisationsmethode und verschiedener Massregeln zum Schutze vor den Bakterien lässt die Behauptung zu, dass die Larvenkulturen wenigstens in den meisten Fällen steril bleiben sollten; das wurde auch bei der Kontrolle des Dr. J. Nikitinsky im bakteriologischen Laboratorium des Landwirtschaftlichen Instituts k onstatiert, aber

1) Es schien nach den bisher angestellten Versuchen, dass solcher Ammoniakzusatz vor der Sterilisation für die Larven nützlich ist und nachherigen Ammoniakzusatz überflüssig macht (wenn eine grosse Menge Eier für den Versuch genommen ist); das letzte aber betrachte ich als noch nicht sicher festgestellt. 
nur einmal und in einem Reagenzglase, in welchem sich nur wenige Larven entwickelten. Gewöhnlich aber bekommt man aus den Nabrungsresten sehr charakteristisehe Bakterienkulturen, von denen einige bei der Kontrolle des Dr. J. Nikitinsky genau untersucht wurden und sich als aus reinen Mikrokokkenkulturen bestehend erwiesen; die Nahrungsreste hatten immer einen sehr deutlichen Ammoniakgeruch, auch in den Fällen, wenn kein Ammoniak hinzugesetzt war. Wenn man eine Verunreinigung oder ungenügende Sterilisation meiner Larvenkulturen annehmen würde, könnte man gar nicht erwarten, eine reine und zwar Mikrokokkenkultur anzutreffen. Das die normalen Eier Bakterien enthalten können, wurde schon früher vermutet ${ }^{1}$ ); in diesem Falle kann das sicher nachgewiesen werden.

Um diese wichtige Erscheinung möglichst ausser jeden Zweifel $\mathrm{zu}$ stellen, haben wir zusammen mit Dr. J. Nikitinsky die Entwicklung der sterilen Eier auf Nährgelatine in den Petri'schen Schalen beobachtet; die auskriechenden Larven gehen lebhaft umher und bedecken die Nährsubstanz mit deutlich sichtbaren Spuren - die letzten enthalten reine Kultur der Mikrokokken.

Wichtiges ergaben auch die wenigen Kulturen, welche einmal aus Versehen ungenügend sterilisiertes Fleisch enthielten: hier waren ausser der Mikrokokkenkultur auch gelatineverflüssigende Mikroorganismen vorhanden, die auch in unausgenutzten Fleischportionen gefunden waren. Die Entwicklung der Larven schritt hier besonders gut vorwärts, und fast alle Larven fühlten sich mehr oder weniger gut; zwei von ihnen verpuppten sich sogar in demselben Reagenzglase; die Puppen waren aber keine normalen, sondern von länglicher Form, welche wie Larvenleichen aussahen und sich bloss durch Farbe, Glanz und Konsistenz von denen unterschieden; sie waren sogar nicht ganz gerade, sondern etwas krumm. Fliegen habe ich indessen keine bekommen, denn beide starben. Solche anormale Fliegenpuppen sind, wie es scheint, bis jetzt noch nicht beschrieben

1) Nämlich von Prof. N. Cholodkowsky für den Fall von Periplaneta.

Bekanntlich gelangen auch bei Hydra viridis einzellige Algen in die Eizellen. Ähnlichen Fall stellt zuweilen Seidenspinner, wenn er die Spaltpilze enthält, welche Krankheit "Pebrine" verursachen; hier dringen die Spaltpilze in die Eier im Körper der Schmetterlinge ein und vermehren sich in den von ihnen entstehenden Raupen. (Vgl. Prof. E. Ziegler, Die Vererbungsiehre in der Biologie. 1905.) 
worden; ich habe sie auch, zwar sehr selten, beim gewöhnlichen Züchten beobachtet, aber aus unbekannter Ursache starben sie jedesmal, ohne Fliegen zu geben, obgleich alles in der dazu nötigen Weise eingerichtet war, während man aus normalen Puppen unter ähnlichen Bedingungen Hunderte von Fliegen enthielt. Ich vermute, dass beim gewöhnlichen Züchten solche Puppen dann öfter entstehen, wenn die Larven in einer Nährsubstanz leben, welche etwas trocken und besonders weniger nahrhaft als gewöhnlich ist (ich habe sie bei Caliphora in einem Gemisch aus Fleisch mit gehackten Pflanzen, bei Musea domestica im Stärkekleister beobachtet.)

Man muss nach allem, was gesagt wurde, vermuten, dass für die Fliegenentwicklung zwei verschiedene Mikroorganismenarten nötig sind, eine von ihnen wird in einer Anzahl der Eier enthalten, die andere kommt aus der Luft.

Ich erkläre solche Erscheinung folgendermassen. Es ist vollständig unrichtig, anzunehmen, dass für die Fliegenlarven jedes verdorbene Fleisch gutes Nährmaterial darbietet; ein kleiner Versuch kann uns darüber aufklären. Verdorbenes Fleisch mit intensivem unangenehmen Geruch war zuweilen bei meinen Versuchen für die Larven gradezu giftig, besonders wenn ich sie in einem gut geschlossenen Behälter züchtete. Die Larven scheinen das Fleiseh für sich sozusagen zu präparieren, indem sie die Entwicklung einiger Bakterien begünstigen, die der anderen aber unterdrücken. Fleischreste von der Larvennahrung riechen sehr stark nach Ammoniak, sonst aber nicht unangenehm und scheinen nicht so tief wie sonst zersetzt zu sein. Wahrscheinlich sind hier gerade Mikrokokken besonders im Spiele! Es ist mir aber nicht gelungen, die Larven sogar in dem Fleische, das von ihnen selbst zersetzt wurde, so gut und schnell gross zu ziehen, wie es in freier Natur geschieht; diejenigen Reagenzgläser, welche solche Kulturen enthielten, waren von den mit frischern Fleische gefüllten Reagenzgläsern nicht $\mathrm{zu}$ unterscheiden. $\mathrm{Ob}$ noch einige technische Fehler vorliegen, oder ob die Bakterien selbst noch eine andere Rolle beim Aufbau des Larvenkörpers spielen, habe ich nicht entscheiden können. Zur Entscheidung dieser Frage ist noch bemerkenswert, dass das Eieralbumin, welches mir im Jahre 1898 doch ziemlich gute Resultate lieferte, von den Larven bei voller Abwesenheit der Bakterien sehr schlecht ausgenutzt wurde - alle Larven starben in den ersten Entwicklungsstadien. Dieses Eieralbumin wurde zuerst durch Tüll filtriert, dann mit dem Fleische, 
welches man als Larvenfutter gebraucht hat, infiziert, mit ein wenig schwacher Ammoniaklösung gemischt und einige Stunden (ungefähr 10) bei Zimmertemperatur stehen gelassen. Es wurde dann durch die $\mathrm{C} \mathrm{h}$ a m be rl a d'sche Filtrierkerze filtriert und mit gewöhnlichen Vorsichtsmassregeln in sterilisierte Reagenzgläser gegossen, welche eine genügende Menge Glaswolle zum Einsaugen enthielten. Warum die in beschriebener Weise dargestellte Nahrung von den Larven so schlecht ausgenutzt wurde, wage ich nicht zu erklären, weil nur wenige Versuche gemacht worden sind.

Ausserdem habe ich Blut, verschiedene von mir dargestellte Eiweisspräparate und Mischungen, als Nährsubstanz verwendet, ohne gute Resultate zu erzielen.

Nach allem, was hier beschrieben ist, muss angenommen werden, dass Ammoniakbildung möglicherweise keine spezifische Erscheinung im Eiweisszerfall der Fliegenlarven darstellt, sondern dass sie von der Bakterientätigkeit herrührt. Das erklärt am einfachsten die von E. Weinland konstatierte Tatsache, dass die Fliegenpuppe keinen Ammoniak ausscheidet und in dieser Beziehung von der Larve so augenfällig abweicht. Es ist auch klar, dass die Fliegen und ihre Larven für manche physiologischen Fragen ausgezeichnete Untersuchungsobjekte darstellen und der Aufmerksamkeit der Forscher warm empfohlen werden sollen. (Manches über Biologie der Fliegen, ibre Physiologie der Sinnen und über die Metboden der Züchtung veröffentlichte ich in folgenden Schriften: Biologische Untersuchungen über Coprophaga (russisch). Mémoires de l'Academ. des Sc. de St. Petersbourg, 1896; Zur Biologie der Coprophaga. Zeitschr. für Allgemeine Entomologie, Itzehoe 1900; 10 Generationen der Fliegen in veränderten Lebensbedingungen. Zeitschr. für Allgemeine Entomologie 1903.)

Es steht mir noch die angenehme Pflicht vor, meine grösste Dankbarkeit Herrn Prof. N. Chudiakow und Herrn Dr. J. Nikitinsky für ihre Ratschläge und liebenswürdige Unterstützung auszusprechen. 